\title{
BUNCH COALESCING STUDIES FOR THE SSC
}

\author{
N. Mahale, Y. T. Yan and J. Ellison \\ Superconducting Super Collider Laboratory* \\ 2550 Beckleymeade Avenue \\ Dallas, TX 75237 USA
}

\begin{abstract}
It may become necessary to enhance the luminosity and increase the bunch spacing in the Superconducting Super Collider (SSC). Here we study the feasibility of bunch coalescing to achieve this. Wc choose the Medium Energy Booster (MEB) at extraction, momentum $200 \mathrm{GeV}$, to perform the operation. In order to properly align the bunches we propose to linearize the RF using the second and third harmonics. Theoretical studies with simulation are presented. The program ESME is used for the simulation.
\end{abstract}

\section{INTRODUCTION}

The fundamental parameters of the collider are, luminosity of $10^{33}$, energy of $20 \mathrm{TeV}$ and bunch spacing of 5 meters. This corresponds to about 2 events per bunch crossing. Thus the detector requirement is to handle at least 2 events every 16 nanoseconds. There are several scenarios which may require a change in the bunch spacing. If the detector dead time cannot handle 16 nanosecond time interval between the bunch crossing it may be necessary to increase the bunch spacing. One way to achieve this is bunch coalescing. The design emittance may not be achieved. Then we could increase the luminosity through bunch coalecsing. If the design energy is not achieved this could be compensated by increasing the luminosity. During the comissioning of the detectors it may be desirable to have a larger bunch spacing. Lastly, luminosity upgrade by itself may be required for the new experiments in future. One way to achieve the higher luminosity is through bunch coalescing if one can accept a higher rate of events per bunch crossing. Here we study the feasibility of this option.

\section{CHOICE OF MACHINE}

The Low Energy Booster is a resonant machine and there fore is out of consideration. To keep the longitudinal emittance, $\epsilon_{l}$, small and power requirements of subharmonic low, it is necessary to reduce the bunch height before coalescing. Due to high energy and low slip factor it would be difficult to reduce the bunch heights in the High Energy Booster or the Collider. If coalescing is done in the $\mathrm{MEB}$, resulting higher longitudinal emittance and larger

* Operated by the Universities Research Association, Inc. for the U. S. Department of Energy under Contract No. DE-AC3589ER40486. bunch spacing will improve the bunch stability, microwave and multibunch, in the HEB and the Collider Thus, the Medium Energy Booster is the most desirable for the operation.

Next we have to choose between the injection and extraction point. The advantage of the injection point is that slip factor here is higher and therefore the procedure will take a shorter time. On the other hand the required rf voltages of subharmonics will be larger. In the MEB the bunches cross the transition energy. The coalescing process inevitably leads to a large longitudinal emittance. Also the new bunches have a greater number of particles and the ratio of peak to average current will be larger. This gives rise to a higher space charge tune shift. If the bunches are coalsced at injection, resulting larger longitudinal emittance and higher space charge tune shift will have a detrimental effect on transition crossing. At extraction the only disadvantage is a somewhat longer time for the procedure. However, the time for the procedure is not unacceptable. Thus, the extraction point in the MEB is the optimum stage for coalescing.

\section{THE BASIC PROCEDURE}

The basic procedure is to turn off the primary RF and turn on the subharmonic. Keep the subharmonic for one quarter of synchrotron period so that all the bunches in the longer bucket align along the energy axes in the phase space. Now turn off the subharmonic and recapture the bunch within the bucket of the primary RF. However, the synchrotron periods for different bunches vary. The bunches near the separatrix move too slowly and a good allignement is not possible. To get a better allignment three things are done. The main RF is adiabatically reduced to reduce the bunch height. The bunches further than $90^{\circ}$ from the synchronous phase $\left(180^{\circ}\right)$ in the subharmonic bucket are assumed to be chopped off. The RF profile between $90^{\circ}$ and $270^{\circ}$ is linearized by superimposing the the second and third harmonics of the subharmonic. These operatons make the bunches align adequately so that the longitudinal emittance and the beam loss during recapture of bunches is kept low. Though we assume that the adiabatic reduction of the bunch height is done after the complete acceleration, it could be done at the tail end of the MEB cycle during the acceleration phase. 


\section{REDUCTION OF RF AMPLITUDE}

Limiting factors with regard to the reduction of the RF amplitude are, the bunch size, time for the process and the multipactoring limit. Since we do not know the multipactoring limit we assume that an effective lower ring voltage can be obtained by paraphasing the cavities. The bucket area must be adequate for the bunch size; we shall provide the bucket at least twice the $95 \%$ emittance.

The remaining important criterion is the time for the process. We define the adiabiticity factor $P$,

$$
P=-\frac{\tau_{s}}{H_{b}} \frac{d H_{b}}{d t},
$$

where, $H_{b}$ is the bucket height and $\tau_{a}$ is the synchrotron period. The adiabiticity factor, $P$, will be kept constant during the reduction of the RF amplitude. Then, the equation for the RF amplitude $\mathrm{V}$ is,

$$
\frac{d t}{d V}=-\frac{\alpha V^{-\frac{3}{2}}}{2 P}
$$

where,

$$
\alpha=\tau \sqrt{\frac{2 \pi \beta^{2} E}{e \eta h}}
$$

where, $\tau$ is the revolution time and $\eta$ is the slip factor. This gives the profile for the RF amplitude and the time required to change the amplitude. Integrating the above equation we get,

$$
t=\frac{\alpha}{P}\left(\frac{1}{\sqrt{V_{f}}}-\frac{1}{\sqrt{V_{0}}}\right)
$$

where $V_{f}$ and $V_{0}$ are the final and the initial RF voltage. We now have to select the adiabaticity parameter $P$, the final RF amplitude and the time. These parameters will be chosen on the basis of simulation results.

\section{LINEARIZATION OF THE RF PROFILE}

The RF system used for accelerating and regular bunching we will refer as primary RF. The subharmonic RF system used for coalescing will be referred as the secondary RF system. Let $A_{1}, A_{2}$ and $A_{3}$ be the amplitudes of the secondary RF and its second and third harmonics. To align all the bunches in the subharmonic bucket we linearize the $\mathrm{RF}$ profile. To linearize we use the second and third harmonics. The scheme we propose is to minimize the integral rms error, the least square fit, in the range of interest beween actual RF profile and the linear profile with a slope $K$. Then we minimize $f\left(A_{i}\right)$, keeping $K$ constant, given by,

$$
f\left(A_{i}\right)=\int_{\pi}^{\phi_{0}} d \phi[V-K(\pi-\phi)]^{2}
$$

where,

$$
V=A_{1} \sin (\phi)+A_{2} \sin (2 \phi)+A_{3} \sin (3 \phi) .
$$

The coefficients $A_{i}$ are to be found by minimizing the error with respect to the linear profile; that is,

$$
\frac{\partial f}{\partial A_{i}}=0 \text {. }
$$

Corresponding to this voltage profile the potential $U(\phi)$ is,

$$
U(\phi)=A_{1} \cos (\phi)+\frac{A_{2}}{2} \cos (2 \phi)+\frac{A_{3}}{3} \cos (3 \phi),
$$

With this potential we then can calculate the period of oscillation. Let $T_{0}$ be the small amplitude period and $T(\phi)$ be the period for the amplitude $\phi$; we define the normalized period $T=\frac{T(\phi)}{T_{0}} . T$ is then given by,

$$
T=\int_{0}^{\phi} d \theta \sqrt{\frac{A_{1}-2 A_{2}+3 A_{3}}{U(\phi)-U(\theta)}} .
$$

\section{THE SIMULATION}

The MEB has $3960 \mathrm{~m}$ circumference, harmonic number 792 and transition gamma of 25.0 . The time available for the operation is about one second. The bunch has $\epsilon_{l}=0.076$ $\mathrm{eVs}$ and the momentum is $200 \mathrm{GeV} / \mathrm{c}$. At extraction, at frequency of $60 \mathrm{MHz}$, the $\mathrm{RF}$ amplitude is $160 \mathrm{kV}$ which produces a bucket with a height $122.5 \mathrm{MeV}$ and an area $2.6 \mathrm{eVs}$. The rms dimensions of the bunch are $9.9 \mathrm{MeV}$ height and $12.95 \mathrm{~cm}$ long. Eleven full buckets and eleven empty buckets alternate in the bunch train. We coalesce 11 bunches which requires $22 n d$ subharmonic. Therefore we need additional RF sources at $2.72 \mathrm{MHz}$, and 5.44 $\mathrm{MHz}$.(the second harmonic) and $8.16 \mathrm{MHz}$.(the third harmonic).

The RF amplitude corresponding to a bucket area of .30 $\mathrm{eVs}$ (this is the least bucket we wish to provide), which is $2.2 \mathrm{kV}$, is not the limiting factor. Thus, the limiting factor will be multipactoring and paraphasing abilility in the presence of errors and beamloading. We use the value of $5 \mathrm{kV}$; this order achieved in the FNAL[1,2,3]. We choose $K=40 \mathrm{kV}$ corresponding to $8 \sigma$ bunch length of 5 meters after the rotation.

The time required to reduce the main $\mathrm{RF}$ amplitude from $160 \mathrm{kV}$ to $5 \mathrm{kV}$ is about $0.158 / \mathrm{P}$ seconds; with $P=0.25$ this time is about 0.63 seconds. The synchrotron tune with the secondary RF systenm is $2.52 \times 10^{-5}$; the revolution time for the machine is $13.21 \mu \mathrm{s}$. Thus, the time required for quarter rotation is $83.30 \mathrm{~ms}$. After the recapture with primary RF we need to decohere the bunches before transfering to the HEB; about 0.1 to 0.2 secondsabout 7 to 15 synchrotron periods- seem to be adequate. Thus the total time for the procedure is $0.8 \mathrm{~s}$ to $0.9 \mathrm{~s}$; This could be further reduced if the primary RF amplitude can be reduced during the acceleration period.

Since the extraction $\gamma_{E}$ is larger than $\gamma_{t}$ we linearize the secondary $\mathrm{RF}$ profile in the range $\left(\frac{\pi}{2}, \frac{3 \pi}{2}\right)$. With $K=40$ $\mathrm{kV}$, the least square fit gives $A_{1}=52.52 \mathrm{kV}, A_{2}=13.84 \mathrm{kV}$ and $A_{3}=2.531 \mathrm{kV}$. Figure 1 shows the linearized profile. 


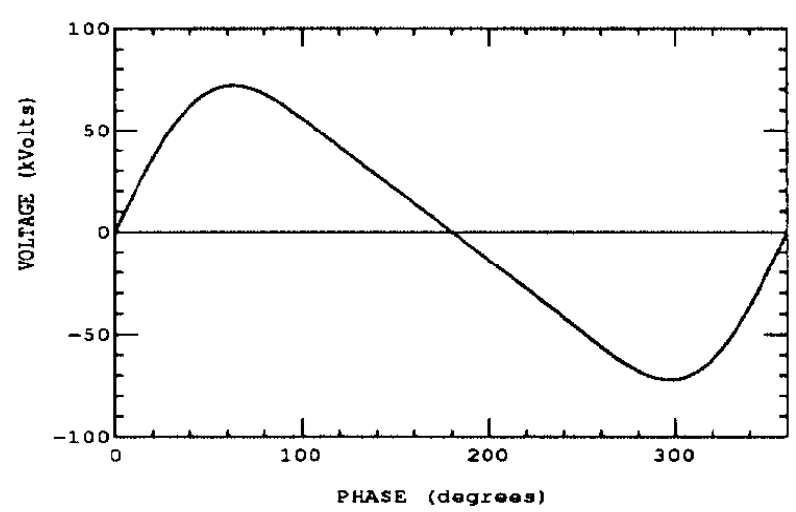

Figure 1. Linearized RF Profile

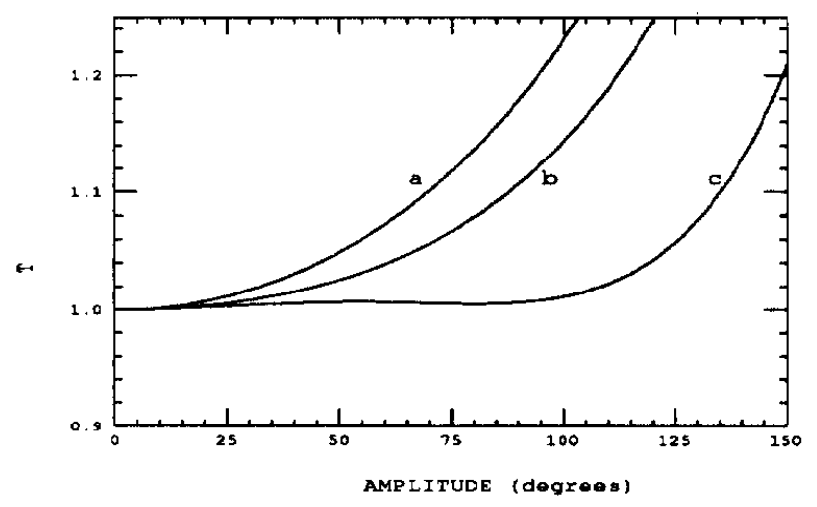

Figure 2. Normalized Period vs Amplitude

In Figure 2 we compare the periods as function of amplitude for three cases: (a) single RF system, (b) linearized with second harmonic and (c) linearized with second and third harmonic.

The simulation of the coalescing was done with the program ESME. Figure 3 shows the eleven bunches aligned in the subharmonic bucket ready to be recaptured in the primary RF bucket. At this point the secondary RF is to be turned off and primary RF turned on. After decohering for 0.20 second we get the final longitudinal emittance to be $4.17 \mathrm{eVs}$ with a beam loss of $0.40 \%$; the rms bunch lenght is $65 \mathrm{~cm}$ and bunch height is $102 \mathrm{MeV}$. During the recapture the bucket parametrs are $V_{c}$ of $800 \mathrm{kV}$, bucket height of $329 \mathrm{MeV}$ and bucket area of $154 \mathrm{eVs}$.

\section{DISCUSSION}

Figure 2 shows that a great improvement is achieved in the constancy of period as function of amplitude due to the inclusion of the third harmonic. We see that the linearization is rather good up to an amplitude of $120^{\circ}-125^{\circ}$. Here we have studied the coalescing of eleven bunches. There is a factor of four loss in luminosity due to chopped bunches; the net gain in luminosity is by a factor $\frac{11}{4}$. In addtion there is a problem of chopping these bunches. By increasing the range of linearization we may coalesce more

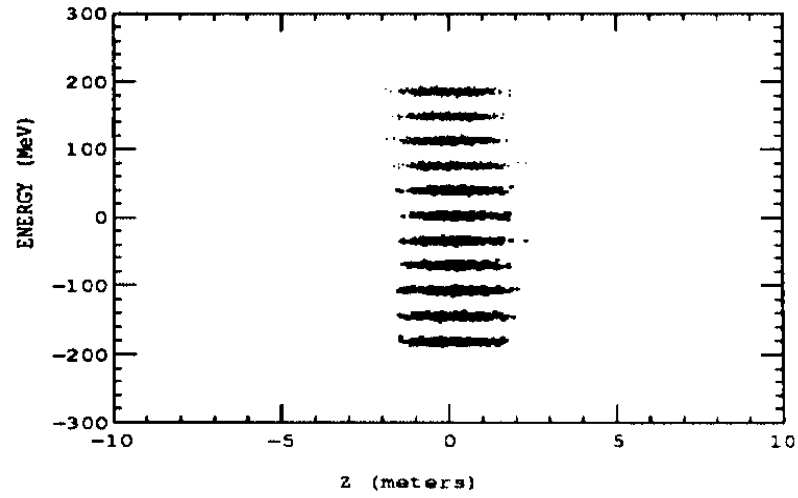

Figure 3. Bunches Aligned to be Recaptured

than half of the original bunches; however, this will require higher RF amplitude and will increase the final bunch height and emittance. For this purpose, if necessary, we also may have to add fourth harmonic.

Alternatively, we could coalesce fewer bunches. Consider the coalescing of three bunches. The problem here is not of alligning the bunches- three bunches can always be aligned-but rather how much the bunch will spread when rotated through quarter synchrotron period; this spread can be reduced by linearization. We need to linearize $120^{\circ}$ about the synchronous phase and can be achieved with three RFs. Here, the gain in the luminosity is 3 ; which is as good as coalescing of eleven bunches. We remark, without giving deatails, that coalesing six bunches culd be done with three harmonics but beyond that we will need additional harmonics. These cases give more luminosity; however, they need more powerful subharmonic RF cavities.

There could be a problem in reducing the primary $\mathbf{R F}$ amplitude, $V_{f}$, to $5 \mathrm{kV}$. To keep the bunch length, which scales as $\left(\frac{V_{f}}{K}\right)^{\frac{1}{4}}$, after rotation we have to scale $\mathrm{K}$ linearly with $V_{f}$; we also need to increase the recapture voltage, $V_{c}$, linearly with $\mathrm{K}$ to keep the beam loss the same.

We thank A. Chao, S. Chen, P. Martin, R. Gerig and M. Syphers for discussions.

\section{REFERENCES}

[1] D. Wildman, P. Martin, K. Meisner and H. W. Miller, "Bunch Coalescing in the fermilab main ring," in 1989 IEEE Particle Accelerator Conference, 1827 (1989).

[2] P. Martin, K. Meisner, and D. Wildman, "Improvements in bunch coalescing in the fermilab main ring," in 1987 IEEE Particle Accelerator Conference, 1028 (1987).

[3] J. Griffin, J. MacLachlan and J. Bridges, "Preparation and Study of Bunches Containing $10^{11}$ protons in the fermilab main ring," in IEEE Trans. Nucl. Sci. NS-28, No. 3, 2037 (1981). 